\title{
Kinematic evidence for feedback-driven star formation in NGC 1893
}

\author{
Beomdu Lim, ${ }^{1,2 \star}$ Hwankyung Sung, ${ }^{2}$ Michael S. Bessell, ${ }^{3}$ Sangwoo Lee, ${ }^{4}$ \\ Jae Joon Lee ${ }^{5}$, Heeyoung $\mathrm{Oh}^{5}$, Narae Hwang ${ }^{5}$, Byeong-Gon Park ${ }^{5,6}$, Hyeonoh Hur ${ }^{7}$, \\ Kyeongsoo Hong ${ }^{5}$, and Sunkyung Park ${ }^{8}$ \\ ${ }^{1}$ Space sciences, Technologies and Astrophysics Research (STAR) Institute, Université de Liége, Quartier Agora, Allée du Août 19c, \\ Bât. B5C, 4000, Liége, Belgium \\ ${ }^{2}$ Department of Astronomy and Space Science, Sejong University, 209 Neungdong-ro, Gwangjin-gu, Seoul 05006, Republic of Korea \\ ${ }^{3}$ Research School of Astronomy and Astrophysics, The Australian National University, Canberra, ACT 2611, Australia \\ ${ }^{4}$ SELab, Inc., 8 Nonhyeon-ro 150-gil, Gangnam-gu, Seoul 06049, Republic of Korea \\ ${ }^{5}$ Korea Astronomy and Space Science Institute, 776 Daedeokdae-ro, Yuseong-gu, Daejeon 305-348, Korea \\ ${ }^{6}$ Astronomy and Space Science Major, University of Science and Technology, 217 Gajeong-ro, Yuseong-gu, Daejeon 34113, Republic of Korea \\ ${ }^{7}$ Daegu National Science Museum, 20, Techno-daero 6-gil, Yuga-myeon, Dalseong-gun, Daegu 43023, Republic of Korea \\ ${ }^{8}$ School of Space Research, Kyung Hee University 1732, Deogyeong-daero, Giheung-gu, Yongin-si, Gyeonggi-do, 17104, Republic of Korea
}

Accepted XXX. Received YYY; in original form ZZZ

\begin{abstract}
$\mathrm{OB}$ associations are the prevailing star forming sites in the Galaxy. Up to now, the process of how $\mathrm{OB}$ associations were formed remained a mystery. A possible process is self-regulating star formation driven by feedback from massive stars. However, although a number of observational studies uncovered various signposts of feedbackdriven star formation, the effectiveness of such feedback has been questioned. Stellar and gas kinematics is a promising tool to capture the relative motion of newborn stars and gas away from ionizing sources. We present high-resolution spectroscopy of stars and gas in the young open cluster NGC 1893. Our findings show that newborn stars and the tadpole nebula Sim 130 are moving away from the central cluster containing two O-type stars, and that the timescale of sequential star formation is about 1 Myr within a 9 parsec distance. The newborn stars formed by feedback from massive stars account for at least 18 per cent of the total stellar population in the cluster, suggesting that this process can play an important role in the formation of $\mathrm{OB}$ associations. These results support the self-regulating star formation model.
\end{abstract}

Key words: stars: formation - stars: kinematics and dynamics - HII regions - ISM: kinematics and dynamics - open clusters and associations: individual (NGC 1893)

\section{INTRODUCTION}

A giant molecular cloud can form a few star-forming complexes with scales of tens of parsecs. The resultant stellar system is a so-called OB association consisting of loose groups of massive OB stars (Ambartsumian 1947). OB associations are considered as the prime sites of star formation and factories of field stars in the Galaxy (Briceño et al. 2007; Brown et al. 1999; Miller \& Scalo 1978). However, their formation process is so far not well established. One reason is the large extent of these objects, and the other is the difficulty of identifying low-mass members from amongst the contaminating foreground and background stars. With the

* Corresponding author, E-mail:blim@uliege.be advent of high-sensitivity instruments at infrared and X-ray wavelengths, a large number of low-mass members have now been identified enabling the mystery of the internal structure and formation process of OB-associations to start to be understood.

OB associations, in general, comprise highly concentrated core clusters and subgroups of low-stellar density (Blaauw 1964; Koenig et al. 2008; Sung et al. 2017). This substructure may reflect their formation process. There are three models addressing the origin of OB associations. From N-body simulations, Kroupa et al. (2001) proposed that about 30 per cent of stars in an embedded cluster can survive as a bound cluster for about a hundred million years while the other stars become a distributed population after the gas is expelled. According to this model, OB associa- 


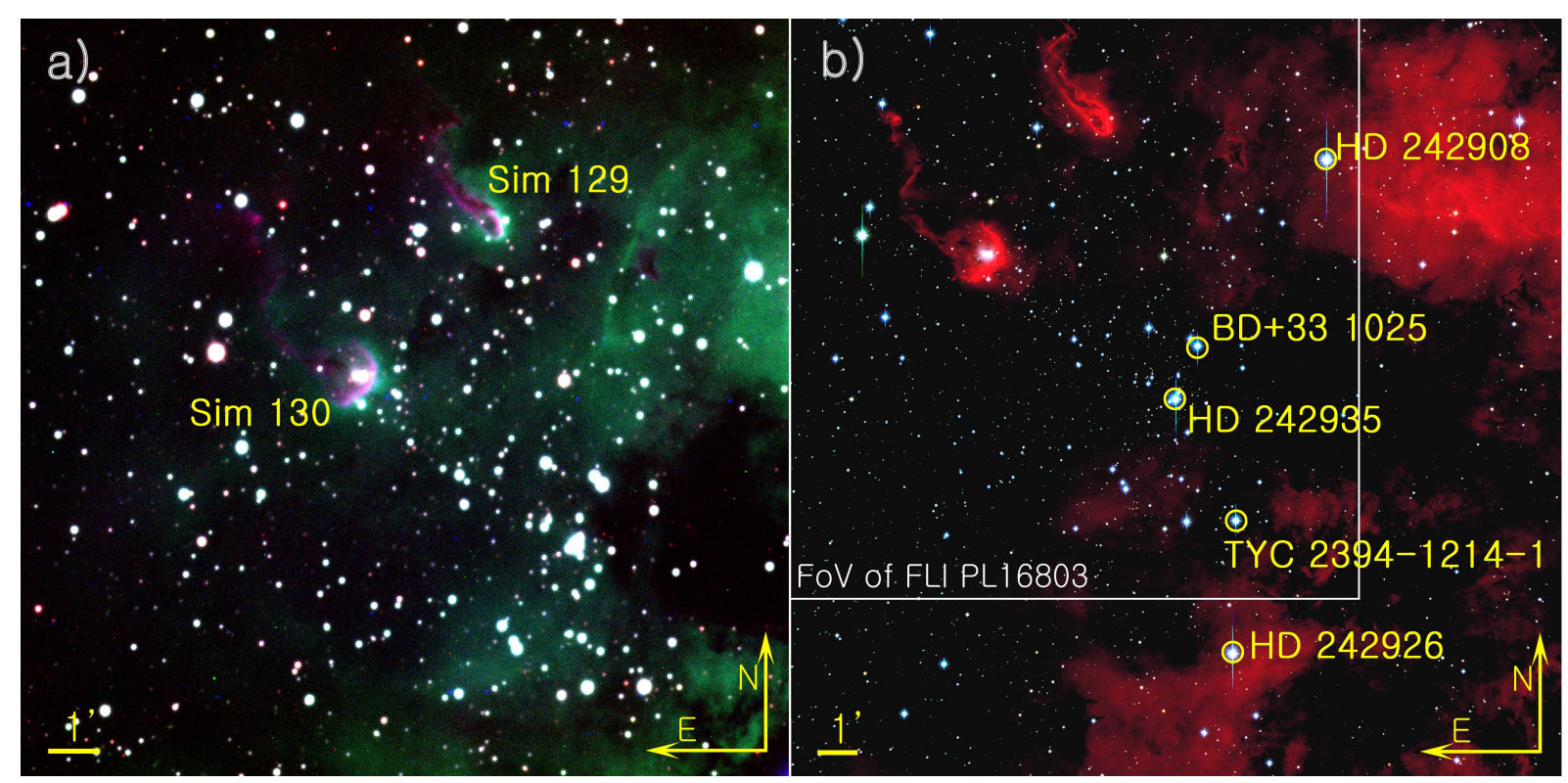

Figure 1. Colour composite images of NGC 1893 (a. blue : H $\beta$, green : [O III] $\lambda 5007$, and red : [S II] $\lambda 6712$, b. blue : $B$, green: $V$, and red : $\mathrm{H} \alpha$ ). The positions of the tadpole nebulae and O-type stars are labelled on each image. The field of view is about $15 ! 8 \times 15 ! 8$ for the FLI PL16803 CCD camera (a) and $18^{\prime} \times 18^{\prime}$ for SNUCam (b). An overlap region between the fields of view of the CCD cameras is outlined by a white solid line.

tions originated from the dynamical evolution of embedded clusters. On the other hand, Clark et al. (2005) claimed that a turbulent unbound giant molecular cloud can form several star clusters containing OB stars on their crossing timescale. In the model, these clusters are expanding having inherited the kinematics of their unbound natal cloud. A clustering of such star clusters independently formed from their natal cloud can have a large spatial distribution over several parsecs as seen in $\mathrm{OB}$ associations. However, neither of these models can explain the substructure within $\mathrm{OB}$ associations that is related to their star formation history.

An alternative model is self-regulating star formation driven by feedback from massive O-type stars (Elmegreen \& Lada 1977). Massive stars can sculpt their natal cloud, creating pillar-like structures and bright-rimmed clouds at the border of $\mathrm{H}$ II regions, as well as ionized gas bubbles (Koenig et al. 2008). A number of sophisticated computer simulations have successfully reproduced these gas structures in ultraviolet radiation fields (Gritschneder et al. 2009a,b; Dale et al. 2012; Haworth et al. 2012). Subgroups of young stellar objects are often found in the vicinity of such gas structures, and moreover, an age sequence among them has been reported from observations (Fukuda et al. 2002; Koenig et al. 2008; Zavagno et al. 2010; Lim et al. 2014, etc.). In addition, the shape of such subgroups appears to be elongated towards the ionizing sources (eg. Getman et al. 2007; Chauhan et al. 2008). These findings are believed to be observational evidence for positive feedback from massive stars. However, caveats from simulations are that all these signs are not necessarily indicative of feedback-driven star formation, and that observationally, stars formed by feedback would be indistinguishable from those spontaneously formed (Dale et al. 2013, 2015).

NGC 1893, a part of the Auriga OB2 association, is ideally suited to validate the self-regulating star formation model because this cluster shows typical signs of feedbackdriven star formation. There are a total of five O-type stars, and a number of young stellar objects have been identified in the vicinity of the tadpole nebulae Sim 129 and 130 (Maheswar et al. 2007; Negueruela et al. 2007). The spatial distribution of the members also shows a highly elongated shape from the cluster centre to the tadpole nebulae (Kuhn et al. 2015), and an age difference between them has been found (Sharma et al. 2007; Maheswar et al. 2007; Pandey et al. 2013).

In Lim et al. (2014), we performed a comprehensive photometric study of NGC 1893. A total of 906 stars were selected as members of NGC 1893 using a colour and magnitude cut method, $\mathrm{H} \alpha$ photometry, and the published catalogues of young stellar objects and X-ray sources (Caramazza et al. 2008, 2012). Based on this membership list, the fundamental parameters of NGC 1893, such as reddening $(\langle E(B-V)\rangle=0.56 \pm 0.08)$, distance $(3.5 \pm 0.3$ kiloparsecs $)$, age (1.5 Myr), and the initial mass function $(\Gamma=-1.3 \pm 0.1)$, were derived by analyzing various photometric diagrams. An age difference of $0.5 \mathrm{Myr}$ was found from comparison of the age distributions of pre-main sequence (PMS) stars in the cluster centre and Sim 130, and we claimed that the age difference is a result of sequential star formation by feedback from the O-type stars in the cluster centre. Measuring the radial velocities (RVs) of the stars and gas would allow us to kinematically identify newborn stars formed by feedback from a coeval population of ionizing sources and to infer their contribution in the formation of an $\mathrm{OB}$ association.

In the present work, we present the high-resolution spectroscopic observations of member stars and ionized gas in NGC 1893. The kinematic substructure of the cluster provides further evidence for a new generation of stars formed by feedback from the first generation of massive stars. The observations and data reduction are described in Section 2. 
The measurement of RVs is addressed in Section 3. The kinematic substructure and age trend within the cluster are investigated with various diagrams in Section 4 and 5, respectively. Multiple sets of Monte-Carlo simulations have been conducted, and the implications are discussed in Section 6 . Finally, we present a summary and conclusions of this study in Section 7.

\section{OBSERVATIONS AND DATA REDUCTION}

\section{$2.1 \quad$ Imaging observations}

We observed a 15 ! $8 \times 15$ ' 8 region of NGC 1893 using the FLI PL16803 CCD camera on the 1-m telescope at Deokheung Optical Astronomy Observatory in Korea on 2016 November 28. The narrow band $\mathrm{H} \beta$, [O $\mathrm{III}] \lambda 5007$, and $\left[\mathrm{S}_{\mathrm{II}}\right] \lambda 6712$ filters were used to study the nebulosity of ionized gas across the cluster. Raw images were subtracted by a master bias image, and subsequently divided by master flat images for given bands. A colour composite image was created by combining the reduced images (see Fig. 1-a).

SNUCam is a $4 \mathrm{k} \times 4 \mathrm{k}$ CCD camera installed on the $1.5-\mathrm{m}$ AZT-22 telescope $(f / 7.74)$ at Maidanak Astronomical Observatory in Uzbekistan (Im et al. 2010). We observed a $18^{\prime} \times 18^{\prime}$ region of NGC 1893 on 2009 January 19 using this camera with the broad band $U B V I$ and narrow band $\mathrm{H} \alpha$ filters. Image pre-processing and photometry of stars are described in our previous work in detail (Lim et al. 2014). A colour composite image was created using $B, V$, and $\mathrm{H} \alpha$ images (see Fig. 1-b).

Early-type stars (O- and B-type) have prominent blue $U-B$ colours, and their reddening and distance can easily be constrained from the $(U-B, B-V)$ diagram and colourmagnitude diagrams, respectively. The member selection of these stars was made by using a colour and magnitude cut method (see Lim et al. 2014 for detail). PMS stars tend to have circumstellar discs and are very active objects. Xray emission and some $\mathrm{H} \alpha$ emission is related to magnetic activity in the young star while strong $\mathrm{H} \alpha$ emission arises from the disc accretion and a warm circumstellar disc emits strongly at infrared wavelengths. We identified $\mathrm{H} \alpha$ emission star candidates from $\mathrm{H} \alpha$ photometry. A list of PMS stars showing mid-infrared excess emission or X-ray emission were taken from the catalogues of young stellar objects (Caramazza et al. 2008, 2012). A total of 906 stars were selected as members of NGC 1893 (Lim et al. 2014).

\subsection{Spectroscopic observations}

Queue scheduled observations of 183 target stars out of 906 cluster members were carried out on 2016 January 26, 27, 28, and 31 with the multi-object high-resolution spectrograph Hectochelle attached to the 6.5-m telescope of the MMT observatory. The spectral resolving power of Hectochelle is about $R \sim 34000$. A total of 240 fibres can be simultaneously used to observe targets and sky background in a single exposure (Szentgyorgyi et al. 2011). The observations of ionized gas were made with the order-separating filter OB 25 transmitting light in the wavelength range of $6475 \AA-6630 \AA$ in a $1 \times 1$ binning mode, while we obtained the spectra of stars in $2 \times 2$ binning mode to achieve a good signal-to-noise ratio using the RV 31 filter covering $5150 \AA$ - $5300 \AA$. Calibration spectra, such as dome flat and ThAr lamp spectra, were also taken, just before and after the target exposure.

All the frames were written in the multiple extension Flexible Image Transport System (FITS). We merged these mosaic images into a single FITS image after overscan correction with IRAF/MSCRED packages. One-dimensional (1D) spectra of targets were extracted using the dofibers task in the IRAF/SPECRED package. Odd- and even-numbered apertures were traced in the dome flat spectra using a spline function fitting method, respectively, and subsequently the 1D spectra of targets and sky were extracted along corresponding apertures. We also corrected for a variation in the pixel-to-pixel response from residuals of the dome flat divided by a high-order spline function. The solutions for the wavelength calibration were determined from ThAr lamp spectra, applied to the dome flat, targets, and sky spectra. The spectra of the targets and sky were flattened by eliminating the blaze function of the echelle spectra from the wavelength-corrected dome flat spectra.

We created a master sky spectrum with a high signalto-noise ratio by median combining the spectra from a few tens of fibres assigned to the blank sky. This master sky spectrum was used to subtract the contribution of airglow to the spectra of stars. The sky-subtracted spectra for the same target were combined into a single spectrum with a better signal-to-noise ratio and normalized by using continuum levels found from a cubic spline interpolation. On the other hand, we combined the spectra of the ionized gas for the same fibre position into a single spectrum.

Spectroscopic observations of six OB stars were carried out with the fibre-fed Bohyunsan Observatory Echelle Spectrograph (Kim et al. 2007, BOES) attached to the 1.8-m telescope at Bohyunsan Optical Astronomy Observatory in Korea in December 2015 and February 2016. We acquired the spectra of the stars with a $300 \mu \mathrm{m}$ fibre whose spectral resolution is about $R=30000$. Calibration frames were obtained either at the end of each night or at the beginning of observations. Pre-processing and extraction of the spectra were conducted with the IRAF/ECHELLE package in almost the same way as the data reduction procedure of Hectochelle. We applied the wavelength calibration obtained from the ThAr lamp spectra to the extracted 1D target spectra, and then those target spectra were flattened and normalized.

The Immersion GRating INfrared Spectrograph (IGRINS) simultaneously obtained the near-infrared $H$ and $K$ band spectra with a high spectral resolution of $R \sim 45000$ (Yuk et al. 2010; Park et al. 2014). This spectrograph was operated with the 2.7-m Harlan J. Smith Telescope at McDonald Observatory of the University of Texas at Austin until the first half year of 2016. Pilot observations of three slit positions on the head of the tadpole nebula Sim 130 was made on 2016 March 2. In the fall of 2016, IGRINS was installed on the 4.3-m Discovery Channel Telescope at Lowell Observatory. We observed six slit positions on the head of Sim 129 and eight positions on that of Sim 130 on 2016 November 18 and 19, respectively. In all the observations, a nod technique, ON (source) - OFF (sky) - ON (source), was applied to the observing sequence for removing the background flux. In order to eliminate a large number of telluric lines, the A0 main sequence stars 136 
Table 1. A summary of observations

\begin{tabular}{|c|c|c|c|c|c|c|}
\hline $\begin{array}{c}\text { UT date } \\
\text { (YY-MM-DD) }\end{array}$ & Target & Telescope & Instrument & Filters & $\begin{array}{l}\text { Exposure time } \\
\text { (seconds) }\end{array}$ & binning \\
\hline \multicolumn{7}{|c|}{ Imaging observations } \\
\hline \multirow{4}{*}{ 2009-01-19 } & & & & $B$ & 20,600 & $1 \times 1$ \\
\hline & & & $18^{\prime} \times 18^{\prime}$ & $V$ & 10,300 & $1 \times 1$ \\
\hline & & & & $I$ & 5,120 & $1 \times 1$ \\
\hline & & & & $\mathrm{H} \alpha$ & 60,600 & $1 \times 1$ \\
\hline \multirow[t]{3}{*}{ 2016-11-28 } & NGC 1893 & $1-\mathrm{m}$ at $\mathrm{DOAO}$ & FLI PL16803 & $\mathrm{H} \beta$ & 1200 & $2 \times 2$ \\
\hline & & & $15 !^{\prime} 8 \times 15 !^{\prime} 8$ & [O III] $\lambda 5007$ & 900 & $2 \times 2$ \\
\hline & & & & {$[\mathrm{S}$ II] $\lambda 6712$} & 600 & $2 \times 2$ \\
\hline \multicolumn{7}{|c|}{ Spectroscopic observations } \\
\hline 2016-01-26 & 14 stars & 6.5-m MMT & Hectochelle & 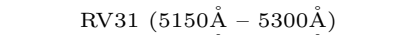 & $3 \times 300$ & $2 \times 2$ \\
\hline 2016-01-26 & 14 stars & & & 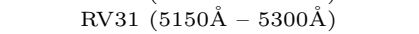 & $3 \times 300$ & $2 \times 2$ \\
\hline 2016-01-26 & 48 stars & & & 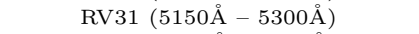 & $3 \times 1200$ & $2 \times 2$ \\
\hline 2016-01-27 & 240 positions & & & OB25 (6475 $-6630 \AA)$ & $3 \times 900$ & $1 \times 1$ \\
\hline 2016-01-31 & 57 stars & & & 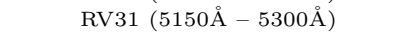 & $3 \times 2700$ & $2 \times 2$ \\
\hline \multirow[t]{5}{*}{$2015-12-17$} & HD 242935 & $1.8-\mathrm{m}$ at $\mathrm{BOAO}$ & BOES & $3800 \AA-9000 \AA$ & $3 \times 1200$ & $1 \times 1$ \\
\hline & $\mathrm{BD}+331025$ & & & & $3 \times 1200$ & \\
\hline & HD 242908 & & & & $3 \times 1200$ & \\
\hline & TYC 2394-1500-1 & & & & $3 \times 1200$ & \\
\hline & TYC 2394-1214-1 & & & & $3 \times 1200$ & \\
\hline $2015-12-27$ & HD 242926 & & & & $3 \times 1200$ & \\
\hline 2016-02-01 & $\mathrm{BD}+331025$ & & & & $3 \times 1200$ & \\
\hline 2016-02-02 & HD 242908 & & & & $3 \times 1200$ & \\
\hline 2016-02-03 & HD 242926 & & & & $3 \times 1200$ & \\
\hline 2016-02-04 & HD 242935 & & & & $3 \times 1200$ & \\
\hline \multirow[t]{3}{*}{ 2016-03-02 } & Sim 130-01 & 2.7-m HJST & IGRINS & $H$ and $K$ bands $(1.45 \mu \mathrm{m}-2.50 \mu \mathrm{m})$ & $3 \times 300$ & $1 \times 1$ \\
\hline & Sim 130-02 & & & & $2 \times 300$ & \\
\hline & Sim $130-03$ & & & & $2 \times 300$ & \\
\hline \multirow[t]{4}{*}{ 2016-11-18 } & Sim 129-00 & 4.3-m DCT & IGRINS & $H$ and $K$ bands $(1.45 \mu \mathrm{m}-2.50 \mu \mathrm{m})$ & $2 \times 300$ & $1 \times 1$ \\
\hline & Sim 129-01 & & & & $2 \times 300$ & \\
\hline & Sim 129-02 & & & & $2 \times 300$ & \\
\hline & Sim 129-05 & & & & $2 \times 300$ & \\
\hline \multirow[t]{8}{*}{ 2016-11-19 } & Sim 130-00 & & & & $2 \times 300$ & $1 \times 1$ \\
\hline & Sim $130-04$ & & & & $2 \times 300$ & \\
\hline & Sim $130-05$ & & & & $2 \times 300$ & \\
\hline & Sim 130-06 & & & & $2 \times 300$ & \\
\hline & Sim $130-07$ & & & & $2 \times 300$ & \\
\hline & Sim 130-08 & & & & $2 \times 300$ & \\
\hline & Sim 130-09 & & & & $2 \times 300$ & \\
\hline & Sim $130-10$ & & & & $2 \times 300$ & \\
\hline
\end{tabular}

Tau, BD $+27716 \mathrm{~B}$, and HR 1558 were observed at almost the same air mass as the tadpole nebulae.

We performed data reduction using the IGRINS Pipeline Package ${ }^{1}$. This pipeline contains basic procedures for data reduction, such as aperture extraction, the subtraction of background emission, bad pixel correction, and wavelength calibration. Subsequently, after correction for telluric lines using the $1 \mathrm{D}$ spectrum of an observed A0 main sequence star with the public tool PLOTSPEC (Kaplan et al. 2017), we stitched together all the echelle spectra to make a two-dimensional relative flux calibrated long spectrum over the $\mathrm{H}$ and $\mathrm{K}$ bands. We summarized our observations in Table 1.

1 The IGRINS Pipeline Package can be download at http://github.com/igrins/plp. doi:10.5281/zenodo.18579

\section{RADIAL VELOCITIES OF GAS AND STARS}

The forbidden line $[\mathrm{N}$ II] $\lambda 6584$ was utilized as a probe of kinematic substructure. This emission line is a prevailing spectral line in $\mathrm{H}$ II regions because its critical density $\left(6 \times 10^{4} \mathrm{~cm}^{-3}\right)$ is two orders of magnitude higher than the typical electron density of the galactic $\mathrm{H}$ in regions (Copetti et al. 2000). Furthermore, photons emitted from the ions are not reabsorbed along the line of sight, and therefore this emission line is a useful tool to study the tomography of the ionized gas. Fig. 2 shows the comparison of [N II] $\lambda 6584$ forbidden line with $\mathrm{H} \alpha$ emission line for the same fibre position.

The profile of the forbidden line $[\mathrm{N}$ II $] \lambda 6584$ for some positions was found to contain a few emission components with different velocities. We obtained the RV of each component using a multiple Gaussian profile fitting method. A total of 1039 emission components were identified from the spectra of 480 fibre positions, and the RVs of 760 components were obtained with errors better than $1 \mathrm{~km} \mathrm{~s}^{-1}$. On the other 

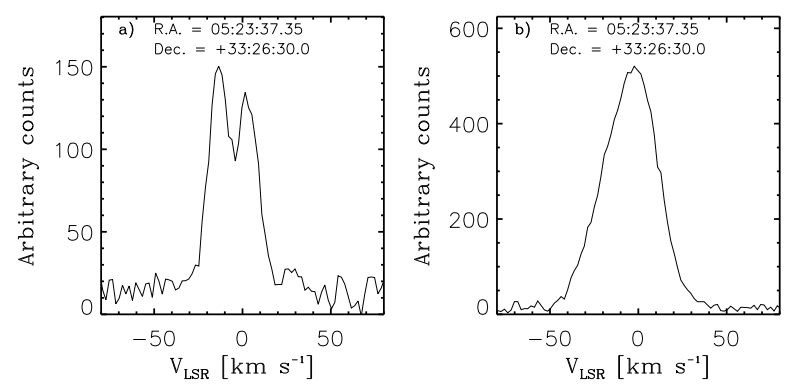

Figure 2. $[\mathrm{N}$ II] $\lambda 6584$ (a) and $\mathrm{H} \alpha(\mathbf{b})$ emission lines from the spectrum for a given fibre position. The forbidden line [N II] $\lambda 6584$ shows a few components with different radial velocities in the line of sight due to absence of absorption column for its spontaneous transition, whereas the $\mathrm{H} \alpha$ line exhibits a simple line profile with a strong single peak at near the systemic velocity of NGC 1893. The processes of reabsorption and emission by prevailing hydrogen atoms with the quantum number $n=2$ in the intracluster medium are responsible for the formation of the observed $\mathrm{H} \alpha$ line profile. Therefore, [N II] $\lambda 6584$ line is a more useful tool to probe the velocity field of ionized gas rather than $\mathrm{H} \alpha$ emission line.

hand, the RVs of warm gas in the tadpole nebulae Sim 129 and 130 were measured from the $1-0 \mathrm{~S}(1)$ emission line of hydrogen molecules at $2.12 \mu \mathrm{m}$. IGRINS provides about 60 near-infrared spectra along the position axis of the slit. We carried out single Gaussian profile fitting to the emission line for given positions and obtained the RVs of Sim 129 and 130 from 342 and 469 positions with measurement errors better than $1 \mathrm{~km} \mathrm{~s}^{-1}$, respectively. All the best fitting solutions were derived with the MPFIT packages (Markwardt 2009).

The spectra of late-type stars in the wavelength range of 5150 - $5300 \AA$ contain a large number of metallic absorption lines as well as the prominent $\mathrm{Mg}$ b triplet at 5167, 5172, and $5183 \AA$. The RVs of those stars can be precisely measured using a cross-correlation technique. We synthesized stellar spectra for the Solar abundance in the wide temperature range of $3800 K$ to $9880 K$ using the spectrum analysis code MOOG (Sneden 1973) and Kurucz ODFNEW model atmospheres (Castelli \& Kurucz 2004). Synthetic spectra of B-type stars in the temperature range of $15000 \mathrm{~K}$ to $25000 \mathrm{~K}$ were obtained from Tlusty model atmospheres (Lanz \& Hubeny 2007). These synthetic spectra were used as template spectra to derive the cross-correlation functions (CCFs) of the observed spectra. Since the spectral types of the observed late-type stars were unknown, we selected the synthetic spectrum with the strongest CCF peak among those of the other synthetic spectra as the template spectrum for an observed star. The velocities corresponding to a CCF peak were determined using RVSAO packages (Kurtz \& Mink 1998). The xcsao task provides the uncertainties of RVs based on the r-statistics as below (Tonry \& Davis 1979):

$r=\frac{h}{\sqrt{2} \sigma_{a}}$

where $h$ and $\sigma_{a}$ are the amplitude of a CCF and the rms from its antisymmetric component, respectively. Then, the measurement error is expressed as $3 w / 8(1+r)$ where $w$ is the full width at half-maximum of the CCF peak (Kurtz $\&$ Mink 1998). The mean error of RVs $\left(\sigma_{\text {err }}\right)$ in this study is about $1.3 \mathrm{~km} \mathrm{~s}^{-1}$. We derived the CCFs of 119 stars, of which eight stars were identified as double-lined spectroscopic binary candidates. The spectra of 22 stars had insufficient signal-to-noise ratios to derive reliable CCFs, while, due to rapid rotation, another $42 \mathrm{~A}$ to B-type stars showed no lines or only a few broad, shallow absorption lines.

O-type and early-B-type stars have a small number of weak metallic absorption lines in their spectra. Hydrogen lines in these stars are, in general, strong broad absorption lines that are often affected by the stellar wind. The crosscorrelation technique is, therefore, less accurate for measuring the RV of O-type stars. However, a handful of helium lines between $4000 \AA$ and $5000 \AA$ can be used to determine their RV since the consistency of RVs from He II $\lambda \lambda 4200$, 4541, He I $\lambda \lambda 4387,4713$, and 4922 has been well tested (Sana et al. 2013). He I $\lambda \lambda 4387,4713$, and 4922 are either too weak or absent in early-O-type stars, and virtually impossible to identify due to severe rotational broadening. Furthermore, these lines can be contaminated by the light from B-type companions. In the present work, He II $\lambda \lambda 4200$ and 4541 were used to measure the RVs of four O-type stars, HD 242908, HD 242926, HD 242935, and TYC 2394-1214-1, while all five helium lines were used for BD +33 1025. The RV of the B0.2V star TYC 2394-1500-1 (Massey et al. 1995) was measured from $\mathrm{He}$ I $\lambda \lambda 4143,4387,4713$, and 4921 as these lines have been used for rapidly rotating B-type stars (Evans et al. 2015). The line centre of the helium lines for the six early-type stars was determined using a Gaussian profile fitting method and subsequently converted to velocity by comparing the wavelength of the line centre with the rest wavelength. We averaged the RVs measured at the same epoch.

Since the O-type stars BD +33 1025, HD 242908, HD 242926, and HD 242935 were observed in two different epochs, the variability of RVs could be checked roughly. BD +33 1025 showed a variation of $12.2 \mathrm{~km} \mathrm{~s}^{-1}$ for 47 days, and this star could be a single-lined spectroscopic binary. The RV of HD 242908 was almost constant for 48 days. This star could be either a single star or long period binary system. A variation of $4.2 \mathrm{~km} \mathrm{~s}^{-1}$ was found for HD 242935 in the centre of the cluster. Since these variations are comparable to the typical uncertainty of RV for O-type stars (a few $\mathrm{km} \mathrm{s}^{-1}$ ), further observations are required to identify binaries among these O-type stars. On the other hand, we found a significant variation of line profiles for He I $\lambda \lambda 4387$, 4713, and 4922 in the spectra of HD 242926. This variation is most likely caused by light from a B-type companion star. Indeed, the RV of this star significantly changed by up to $61.8 \mathrm{~km} \mathrm{~s}^{-1}$ over 39 days. Hence, HD 242926 is a certain double-lined spectroscopic binary. All the double-lined spectroscopic binary candidates identified in this study were not used for further analysis. The RV measurements from this work are available in the electronic tables (Table 2 and 3 ) or from the author (BL).

2 The animation of this figure can be accessed through the links http://starburst.sejong.ac.kr/data/fig-3b_ani. gif and http://starburst.sejong.ac.kr/data/N1893_ppv_ul. gif 

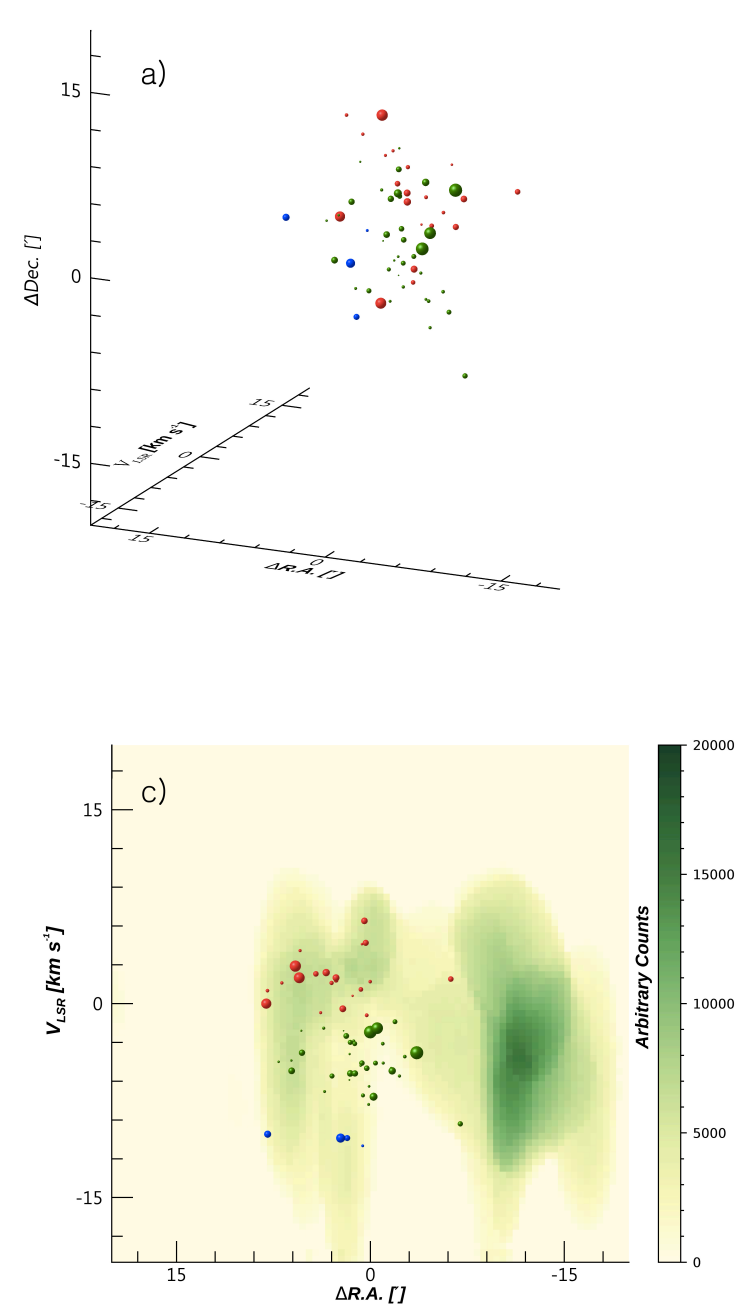
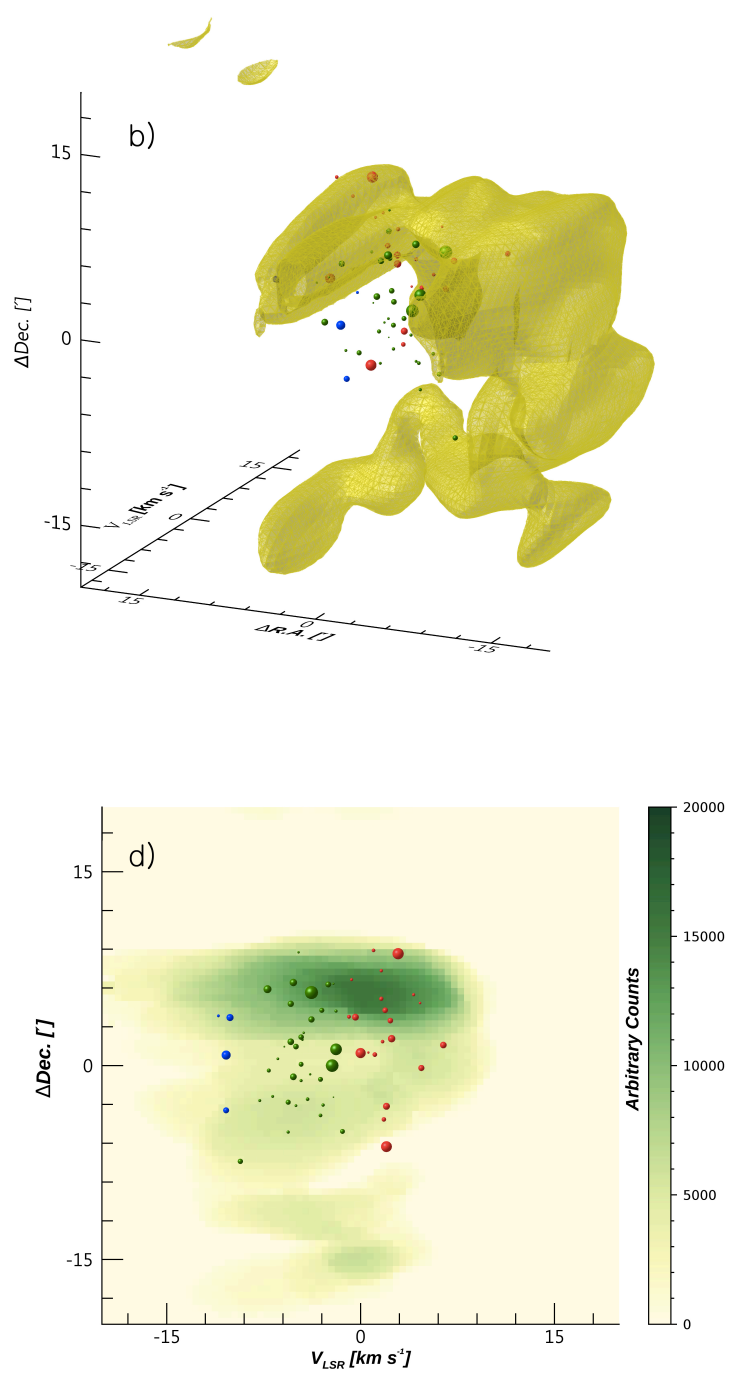

Figure 3. Position-position-velocity diagrams (a and $\mathbf{b}^{2}$ ) and position-velocity diagrams (c and $\left.\mathbf{d}\right)$ of NGC 1893 . Isosurface and colour contours represent the distribution of ionized gas traced by the forbidden line $[\mathrm{N} \mathrm{II}] \lambda 6584$, where the unit of the counts is electrons $\left(e^{-}\right)$. Spheres denote the stars in the cluster, and their size is proportional to the brightness of individual stars. Blue, green, and red colours represent three different groups in the radial velocity range of $V_{\mathrm{LSR}}=-19 \mathrm{~km} \mathrm{~s}^{-1}$ to $-10 \mathrm{~km} \mathrm{~s}^{-1}$, $-10 \mathrm{~km} \mathrm{~s}$ to $-1 \mathrm{~km} \mathrm{~s}-1$, and $-1 \mathrm{~km} \mathrm{~s}^{-1}$ to $8 \mathrm{~km} \mathrm{~s}^{-1}$, respectively. The position of objects is relative to the O-type star HD 242935 (R.A. $=05^{\mathrm{h}} 22^{\mathrm{m}} 46 .{ }^{\mathrm{s}} 5$, Dec. $\left.=+33^{\circ} 25^{\prime} 11^{\prime \prime}, \mathrm{J} 2000\right)$.

\section{KINEMATIC SUBSTRUCTURE}

The colour-composite images of NGC 1893 show the spatial distribution of hot ionized gas and stars (Fig. 1). A total of five O-type stars are distributed in a north-south direction, two of which are located in the cluster centre. The intracluster medium is filled with hot ionized gas as seen in the [O III $\lambda 5007$ image. The tadpole nebulae Sim 129 and 130 facing towards the two O-type stars in the cluster centre are glowing in the narrow band $\mathrm{H} \alpha, \mathrm{H} \beta$, and [S II] $\lambda 6712$ filter images as shock heated gas-flows surround these nebulae.

We present two and three-dimensional views of this cluster in position-velocity space as shown in Fig. 3. In order to display the distribution of the ionized gas in the diagrams, a data cube made of $80 \times 80 \times 80$ volume cells was cre- ated by linearly interpolating the scattered data values to points in a regularly sampled volume. In this procedure, the observed parameters, such as the fibre positions (right ascension and declination), the velocity profiles of the $[\mathrm{N} \mathrm{II}]$ emission line, and the counts $\left(e^{-}\right)$which were irregularly scattered in a three-dimensional space were interpolated to a regular grid cube by applying Delauney triangulation technique. The two-dimensional position-velocity diagrams were obtained by the sum of the counts along one position axis (either right ascension or declination) in the data cube.

The isosurface shows the distribution of the ionized gas that surrounds the whole cluster like a bubble (Fig. 3-b). Such a gas bubble appears open towards observers in the position-velocity diagrams (c and d). Its asymmetric shape is attributed to the inhomogeneous distribution of matter. 

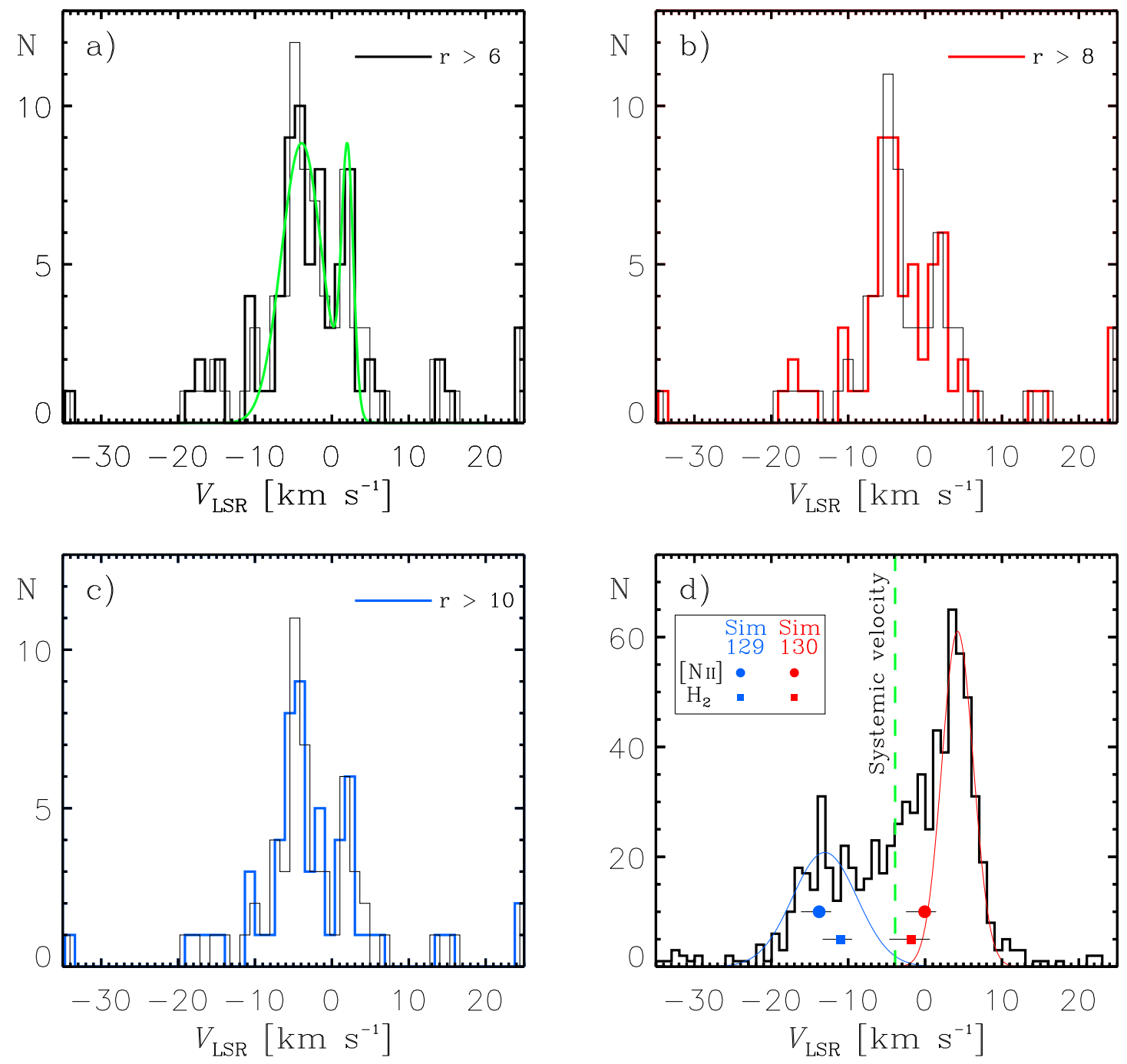

Figure 4. Radial velocity distributions of stars $(\mathbf{a}-\mathbf{c})$ and gas $(\mathbf{d})$. Black, red, and blue histograms represent the radial velocity distributions for subsets of stars with r-statistics larger than 6,8 , and 10 , respectively. The bin size is $1.3 \mathrm{~km} \mathrm{~s}^{-1}$, equivalent to the mean measurement error. To avoid the binning effect, we also overplotted histograms (thin solid line) shifted by $0.65 \mathrm{~km} \mathrm{~s}^{-1}$. The radial velocity distribution of stars was fitted by a double-Gaussian profile. The histogram (d) exhibits the radial velocities of ionized gas traced by the forbidden line $[\mathrm{N} \mathrm{II}] \lambda 6584$. The dashed line represents the systemic velocity of the cluster. The mean velocities of each bubble obtained from a Gaussian profile fitting method are about $-13.0 \pm 1.4$ and $4.2 \pm 0.1 \mathrm{~km} \mathrm{~s}^{-1}$, respectively. The circles show the mean velocities of the ionized component in the tadpole nebulae Sim 129 (blue) and 130 (red), while the squares denote those of their warm components measured from the $1-0 \mathrm{~S}(1)$ emission line of hydrogen molecules. The error bars indicate the minimum and maximum values of their radial velocities.

The fact that the stars and ionized gas coexist in positionvelocity space, indicates that they are physically associated components in NGC 1893. The ionizing source is O-type stars rather than either supernova explosions of the most massive stars, or violent activities arising from compact objects, because NGC 1893 is too young to produce the sources that could cause such energetic events.

The RVs of the stars show a bimodal distribution (Fig. 4-a). The possibility that the observed distribution was from a single Gaussian distribution is less than 10 per cent according to a Kolmogorov-Smirnov test. The bimodality is conserved even when the r-statistics is changed or when the histograms were shifted by the half size of the adopted velocity bin. We also investigated the histograms by changing the bin sizes of $n \sigma_{\text {err }}$ (where $n=0.5,1.0,1.5,2.0$, and 2.5). The components of the subgroup were clearly seen in the histograms (with confidence levels at least two times higher than the Poisson noise of the histogram of the central cluster) until with the bin size of $2.5 \sigma_{\text {err }}$ they merged into that of the central cluster. Hence it is certain that there are, at least, two groups of stars with different velocity fields in the line of sight.

We fitted a double-Gaussian profile to the observed RV distribution as seen in Fig. 4-a. The main peak in the histogram indicates the systemic velocity of NGC 1893. The systemic velocity of the cluster was estimated to be $-3.9 \pm 0.4 \mathrm{~km} \mathrm{~s}^{-1}$. The dispersion of the systemic velocity is about $2.6 \mathrm{~km} \mathrm{~s}^{-1}$. Given a mean measurement error of 1.3 


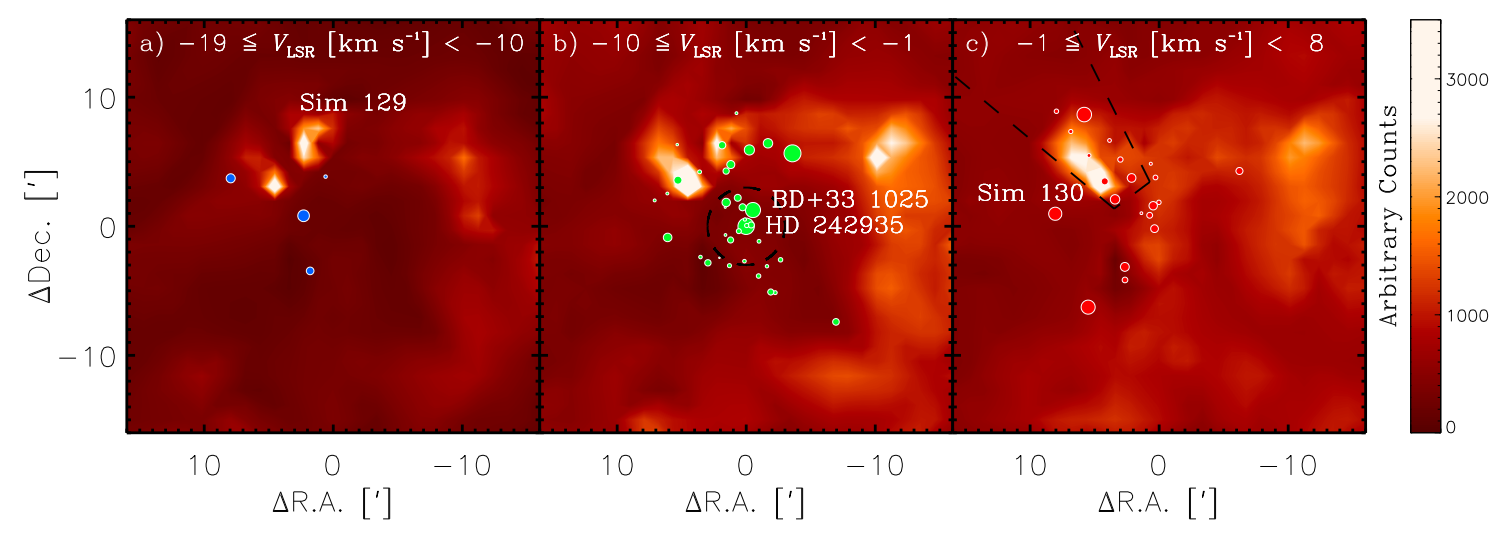

Figure 5. a - c , integrated channel maps of ionized gas across NGC 1893. These maps were obtained by integrating all the counts of the forbidden line [N II] $\lambda 6584$ within the given velocity ranges. Stars in the same velocity ranges as the ionized gas are also plotted. The size of circles is proportional to the brightness of individual stars. The coordinates relative to the cluster centre are the same as those in Fig. 3. The circle (dashed line) in $\mathbf{b}$ represents the size of the central cluster inferred from the radial surface density profile of stars $\left(3^{\prime}\right)$, and the kinematic subgroup is confined by a region outlined by dashed lines in $\mathbf{c}$.

$\mathrm{km} \mathrm{s}^{-1}$, as well as the contribution of binaries with small velocity variations, the intrinsic velocity dispersion is likely to be smaller than $2.3 \mathrm{~km} \mathrm{~s}^{-1}$. The RV of the subgroup corresponding to the secondary peak is about $+2.0 \pm 0.3 \mathrm{~km}$ $\mathrm{s}^{-1}$, and its dispersion is about $0.8 \mathrm{~km} \mathrm{~s}^{-1}$ which is smaller than the measurement error. The small dispersion may be attributed to the small number of sample stars.

The RV distribution of the ionized gas also exhibits a non-Gaussian distribution (Fig. 4-d), as can be expected from its bubble structure. The well-defined systemic velocity from the RV distribution of stars almost equally divides the gas bubble into a near side part and a far side part. The component with a low-amplitude at $-13.0 \mathrm{~km} \mathrm{~s}^{-1}$ is indicative of a small amount of material on the near side of the bubble. On the other hand, the component with a high-amplitude at $4.2 \mathrm{~km} \mathrm{~s}^{-1}$, corresponds to material on the far side of the bubble where ionization fronts proceed into the remaining molecular cloud. The relative RV of each peak compared to the systemic velocity indicates the ionized bubble has an expansion velocity of $8.1-9.1 \mathrm{~km} \mathrm{~s}^{-1}$ from the cluster centre. The mean RVs of the ionized gas and warm hydrogen molecules in the tadpole nebulae show that they lie at different parts of the ionized gas bubble.

Sim 129 is a clump in the direction of the near side of the bubble (Fig. 4-d). No star formation has taken place in Sim 129 given that no star is kinematically associated with the nebula (Fig. 5-a). The largest rim of ionized gas seen in the integrated channel map Fig. 5-b is the section of a bubble. If the gas bubble has been being expanding at a mean velocity of $8.6 \mathrm{~km} \mathrm{~s}^{-1}$ for $1.5 \mathrm{Myr}$ at 3.5 kiloparsecs, the size of the rim should be about $26^{\prime}$ in diameter. This estimate is in reasonable agreement with the size of the observed bubble. Stars in the same velocity range are distributed across the whole region. Since HD 242935 and BD +33 1025 in the cluster centre have velocities within the systemic velocity range, they are major contributors to the creation of the central cavity and the tadpole nebulae. The most prominent feature in Fig. 5-c is the distribution of stars correlated with the head-tail structure of Sim 130 (see the region outlined by dashed lines). This kinematic substructure is unlikely to result from the dynamical evolution because the age of the cluster is 2.5 times younger than the crossing time of the stars $\left(\frac{8.9 \mathrm{pc}}{2.3 \mathrm{~km} \mathrm{~s}^{-1}} \sim 3.8 \mathrm{Myr}\right.$, see Section 6$)$. We find, therefore, that this group is spatially and kinematically associated with Sim 130.

A velocity variation of $2.7 \mathrm{~km} \mathrm{~s}^{-1}$ was found along the head-tail structure of Sim 130 (see Fig. 6-a). The ionized gas tail has almost the same velocity field as that of the subgroup, whereas its head is moving away more slowly than its tail from the cluster centre. Given that neither remarkable acceleration nor deceleration was found in the RVs of the stars in the subgroup, the velocity variation probably does not represent the acceleration history of the ionized gas bubble. Irradiation on an inhomogeneous molecular cloud is likely responsible for the formation and evolution of Sim 130 (Gritschneder et al. 2009b).

\section{AGE TREND}

The subgroup in the vicinity of Sim 130 is expected to be younger than the central cluster if star formation was triggered by feedback. In order to confirm the age difference between the central cluster and the subgroup, the age distribution of PMS stars was investigated using our large photometric sample. Their age was inferred from the HertzsprungRussell diagram by comparing their effective temperature and bolometric magnitude with those of evolutionary models (see Lim et al. 2014 for detail). PMS stars with a photometric error smaller than 0.1 mag were used to obtain a reliable age distribution. The errors in the visual magnitude, $V-I$ colour, reddening correction, distance modulus, and bolometric correction were propagated. Apart from the errors in observations and calibrations, the duration of a star-forming event can cause an additional broadening of the age distribution. While there could be a complicated star-formation history in the whole region, the star-formation timescale in a small region, such as the central cluster, may be negligible compared to that of the whole region. We, therefore, restricted our sample to PMS stars within the radius of the central cluster $\left(3^{\prime}\right)$ inferred from the radial surface density profile of stars. 


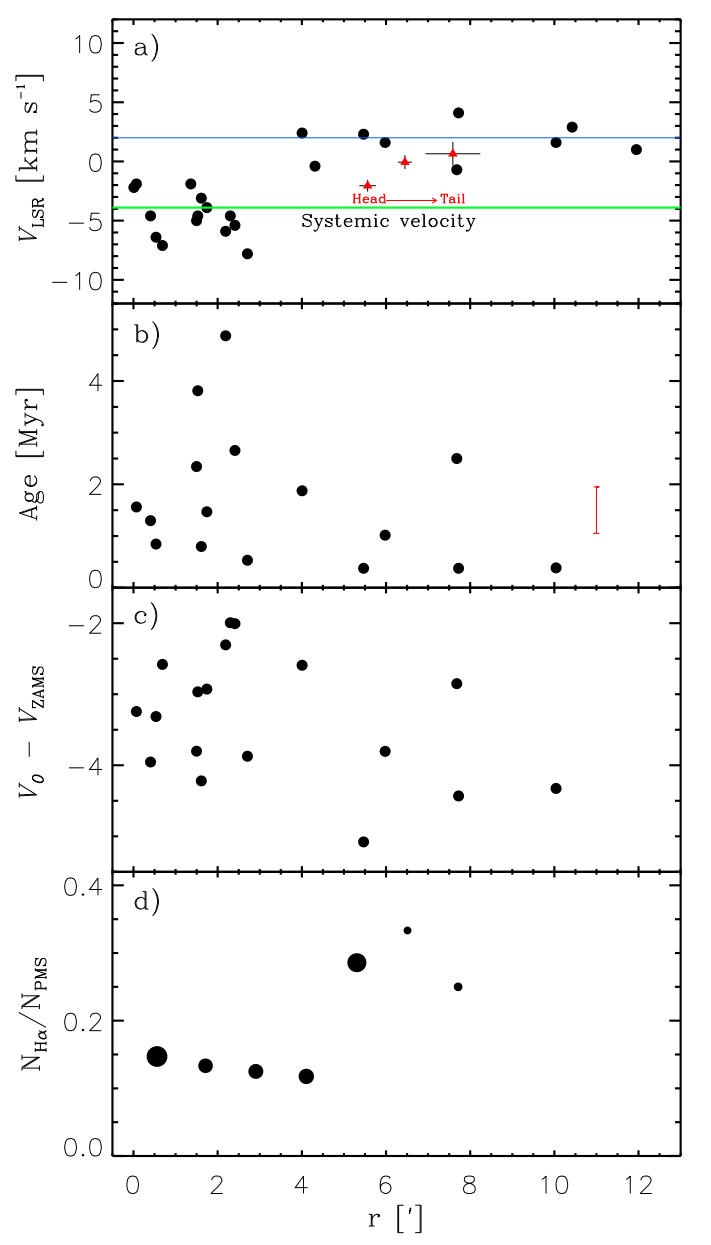

Figure 6. Radial variation of the properties of pre-main sequence stars in the regions outlined by dashed lines in Fig. 5-b and c. (a) The radial velocity distribution of the stars. Blue thin and green thick solid lines represent the mean radial velocity of newborn stars and the systemic velocity of the cluster, respectively. Red triangles shows radial velocity variation along the head-tail structure of Sim 130. (b) The age distribution of the stars inferred from the evolutionary models for pre-main sequence stars (Siess et al. 2000). The error bar denotes the size of the typical error. (c) The brightness distribution of pre-main sequence stars. $V_{0}-V_{\mathrm{ZAMS}}$ represents the difference of visual magnitude between the pre-main sequence stars and the zero-age main-sequence relation at given colours. (d) The variation of the fraction of $\mathrm{H} \alpha$ emission stars. The size of the circles are proportional to the number of pre-main sequence stars. See the main text for details.

The derived age distribution showed a broad asymmetric Gaussian profile with a strong peak at $1.5 \mathrm{Myr}$ corresponding to the age of the central cluster. Given that the age distribution is almost the same as the distribution of the propagated error, the dispersion of the age distribution represents the error in age estimation. We conducted a skew Gaussian profile fitting to this age distribution. The typical error in age was estimated to be 0.9 Myr from the dispersion $(\sqrt{\text { variance }})$ of the best-fit profile. However, the age error of individual stars could be larger than the typical error due to the imperfect correction of differential reddening and systematic uncertainties in evolutionary models for 1.5 - 2 solar-mass PMS stars (Sung et al. 2004).
We plotted the age of the PMS stars within two regions outlined by dashed lines in Fig. 5-b and $\mathbf{c}$ with respect to the distance from the cluster centre (Fig. 6-b). Since the age distribution has a long tail from $5 \mathrm{Myr}$ towards old age, PMS stars younger than that age were used to seek a clear age gradient. Interestingly, in the subgroup of stars in the direction of Sim 130, the age of the PMS stars tend to be younger than that of stars in the cluster centre. This age difference between stars in the vicinity of Sim 130 and the cluster centre has been reported in other work (Sharma et al. 2007; Maheswar et al. 2007; Pandey et al. 2013) as well as in our previous work (Lim et al. 2014). However, since the age difference is comparable to the typical error, this result does not guarantee the robustness of the age difference between them.

We tried to test two different age indicators. The luminosity of PMS stars decreases as they contract along their Hayashi tracks, and therefore younger PMS stars are brighter than older stars at a given temperature. The extinction of individual PMS stars in visual magnitude $V$ was corrected using the reddening map (Lim et al. 2014), and then the extinction-corrected visual magnitude $\left(V_{0}\right)$ was compared with that $\left(V_{\mathrm{ZAMS}}\right)$ of the zero-age main sequence relation at given colours (Sung et al. 2013). Fig. 6-c shows the radial variation of $V_{0}-V_{\mathrm{ZAMS}}$. The lower the value, the younger the star. Star ID 6091 (R.A. $=05^{\mathrm{h}} 23^{\mathrm{m}} 24 .^{\mathrm{s}} 72$, Dec. $=+33^{\circ} 34^{\prime} 05 .^{\prime \prime} 8$ ) was found near the main sequence band although it was identified as a Class II object (Caramazza et al. 2008). This star may have a nearly edge-on disc as did those found in the young open cluster NGC 2264 (Sung et al. 2009). We excluded this star in this analysis. The median values of $V_{0}-V_{\mathrm{ZAMS}}$ for the central cluster and the subgroup are -3.0 and -3.8 , respectively. The subgroup seems to be slightly younger than the central cluster.

Since the number of strong $\mathrm{H} \alpha$ emission stars which can be identified by $\mathrm{H} \alpha$ photometry rapidly declines as a function of time, the fraction of $\mathrm{H} \alpha$ emission stars can be used as an age indicator. Seven circular regions with the same area (0.79 square arcminutes) were chosen along the line joining the central cluster and Sim 130. The number of $\mathrm{H} \alpha$ emission stars and that of all the identified PMS stars were counted within given areas, respectively, and then the fraction of $\mathrm{H} \alpha$ emission stars were computed for each area. Fig. 6-d shows that the fraction in the vicinity of Sim 130 is about two times higher than that in the central cluster. Although $\mathrm{H} \alpha$ emission can vary with time depending on the degree of accretion activity, such a distinctive difference between the two regions cannot be explained by variability. These results are indicative of an age difference and support the fact that the subgroup associated with Sim 130 is a new generation of stars formed in the expanding gas due to feedback from the O-type stars.

The Taurus-Auriga $\mathrm{T}$ association covers a $40 \times 40$ square parsec area and is an ideal comparison field because, low-mass star formation is dominant in the absence of massive O-type stars. The subgroups of young stars show substructure which is considered to be inherited from the filaments of their natal clouds (André et al. 2014) and share almost the same kinematic properties with each other (Bertout \& Genova 2006). The stars of the subgroups do not show any systematic difference (Palla \& Stahler 2002) within $4 \mathrm{Myr}$, and therefore the formation of the subgroups 

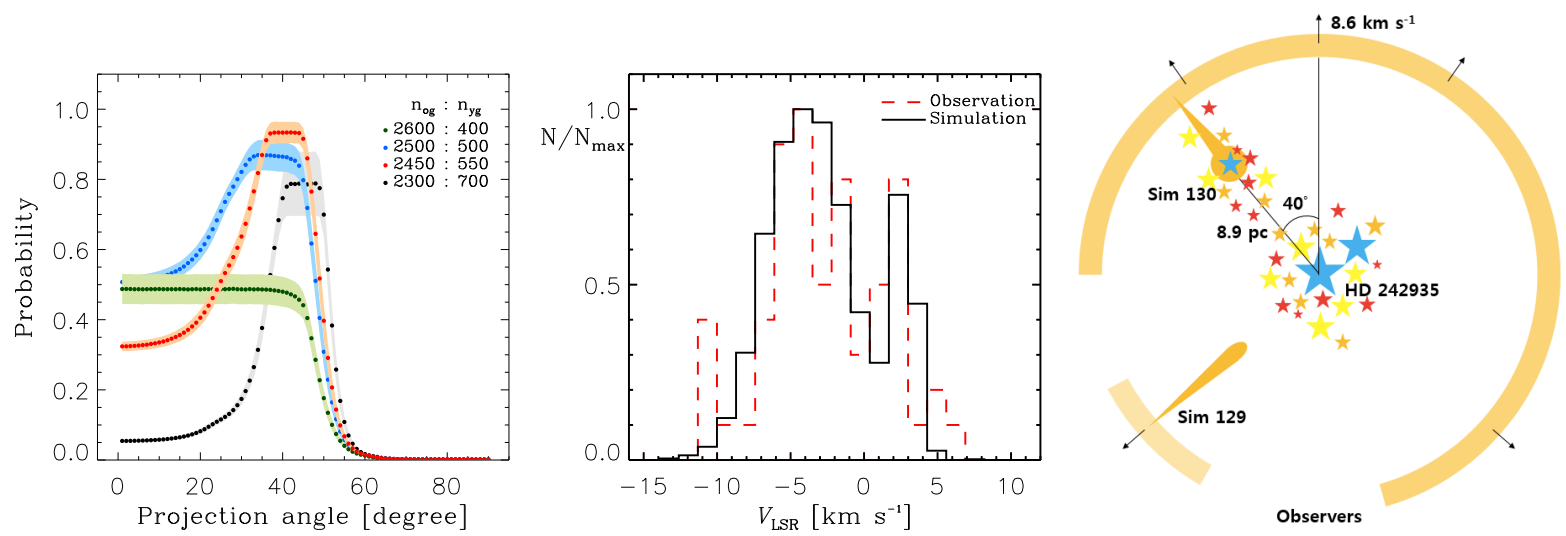

Figure 7. Probability functions of projection angles to the line of sight (left), comparison of the best model with the observed distribution (middle), and schematic figure overlooking NGC 1893 (right). The projection angle is defined as the angle between the kinematic subgroupHD 242935-the line of sight. The mean probabilities were plotted by dots, and their $1 \sigma$ errors were shown by shaded regions. Different number ratios between old and young groups were applied for the simulations as shown by different colours. The best solution with the highest confidence level is the model cluster adopting a number ratio of $2450: 550\left(n_{\mathrm{og}}: n_{\mathrm{yg}}\right)$ at a projection angle of about $40 \pm 2^{\circ}(\mathrm{s}$. d.). The schematic figure exhibits the internal structure of the cluster reconstituted from stellar and gas kinematics. A huge ionized gas bubble is expanding at $8.1-9.1 \mathrm{~km} \mathrm{~s}^{-1}$ (on average $8.6 \mathrm{~km} \mathrm{~s}^{-1}$ ) away from the central O-type stars. Dense parts of a molecular cloud can remain as gas pillars as the massive stars blow out the remaining gas. Depending on the local gas density distribution, some of the dense pillars compressed by ionization and shock fronts will form a new generation of stars.

in the Tau-Aur T association seems to have occurred at almost the same time. The kinematic and photometric properties of stars in NGC 1893 are far different from those of stars in the Taurus-Auriga $\mathrm{T}$ association. Hence, the newborn stars in the subgroup with a receding velocity from the central cluster are not stars spontaneously formed at a recent epoch.

\section{CONTRIBUTION OF THE NEW GENERATION OF STARS}

NGC 1893 is composed of about 3000 stars according to the intrinsic number of the cluster members inferred from its initial mass function (Lim et al. 2014), and the members are divided into at least two groups with different velocity fields; an old group with the systemic velocity of NGC 1893 and a young group. The young group is moving away from the old group at a specific projection angle, where the projection angle is defined as the angle between the young group-HD 242935-the line of sight (see Fig. 7). The stars in the young group will then have RVs as below:

$V_{\mathrm{obs}}=V_{\mathrm{sys}}+V_{\mathrm{exp}} \times \cos \theta_{\text {pro }}$

where $V_{\text {sys }}, V_{\exp }$, and $\theta_{\text {pro }}$ are the systemic velocity of NGC 1893 , the expanding velocity of the ionized gas bubble, and a projection angle, respectively. Here, the ionized gas bubble was assumed to be isotropically expanding from the central cluster at $8.6 \mathrm{~km} \mathrm{~s}^{-1}$ which is the mean expanding velocity of the $\mathrm{H}$ II bubble. Hence, the observed RV distribution of stars depends on the geometric distribution of stars within NGC 1893. In addition, the relative amplitudes of the observed RV distribution between the old and young groups are related to their number ratio. We performed multiple sets of simulations to obtain the geometry of NGC 1893 and the number ratio.

Artificial stars in the old group were set to have RVs drawn from a Gaussian distribution with the systemic velocity of $-3.9 \mathrm{~km} \mathrm{~s}^{-1}$ and the observed velocity dispersion of $2.6 \mathrm{~km} \mathrm{~s}^{-1}$ using a Monte-Carlo method. The RVs of artificial stars in the young group were obtained by substituting various projection angles from $0^{\circ}$ to $90^{\circ}$ with a $1^{\circ}$ interval to the Eq. 2, and the velocity dispersion of $0.8 \mathrm{~km}$ $\mathrm{s}^{-1}$ was applied to the RV distributions of this group. Arbitrary number ratios between the old and young groups were assumed to be $n_{\mathrm{og}}: n_{\mathrm{yg}}=2300: 700,2400: 600,2500: 500$, 2600:400 and 2700:300. A given run for each projection angle and number ratio was repeated 10,000 times, and the resultant distributions were compared with the observed RV distribution, where stars satisfying two criteria; 1) r-statistics $>6,2)-12<V_{\mathrm{LSR}}\left[\mathrm{kms}^{-1}\right]<12$, were used. The probabilities, that represent similarity between the RV distributions from observation and simulations, were computed using a Kolmogorov-Smirnov test, and these were averaged for the same parameter setup. The highest mean probability among these runs was found between $n_{\mathrm{og}}: n_{\mathrm{yg}}=2400: 600$ and 2500:500. We made fine adjustments by changing the number ratios to $n_{\mathrm{og}}: n_{\mathrm{yg}}=2440: 560,2450: 550$, and 2460:540. The model cluster adopting $n_{\mathrm{og}}: n_{\mathrm{yg}}=2450: 550$ well reproduced the observed RV distribution at $40 \pm 2^{\circ}$ (s. d.) with a 93 per cent confidence level (Fig. 7).

We obtained the proper distance of $8.9_{-0.35}^{+0.39}$ parsecs between the head of Sim 130 and the O-type star HD 242935 from the projected distance $(5.7$ parsecs) and the projection angle using a trigonometric function. The timescale of feedback-driven star formation is about $1 \mathrm{Myr}$ assuming a mean expanding velocity of $8.6 \mathrm{~km} \mathrm{~s}^{-1}$. The triggered population accounts for about 18 per cent of all the cluster members according to the number ratio used in the best model. This contribution seems to be a lower limit because we did not take into account the motion perpendicular to the line of sight. Our results suggest that feedback from massive stars in a core cluster can play an important role in building-up the stellar content of $\mathrm{OB}$ associations. 


\section{SUMMARY AND CONCLUSION}

In the present work, we tested the self-regulating star formation model proposed by Elmegreen \& Lada (1977) using high resolution spectroscopy of stars and gas in the young open cluster NGC 1893. The results are summarized as below:

(i) Narrow band images show that hot gas fills the intracluster medium as seen in the [O III] $\lambda 5007$ filter image, and that shock-heated gas surrounds the tadpole nebulae Sim 129 and 130.

(ii) The three-dimensional position-position-velocity and position-velocity diagrams show that an ionized gas bubble surrounds the cluster members, and that the gas and stars are globally associated with each other. The systemic velocity of NGC 1893 is about $-3.9 \mathrm{~km} \mathrm{~s}^{-1}$ and the ionized gas bubble is expanding from the cluster centre at $8.1-9.1 \mathrm{~km}$ $\mathrm{s}^{-1}$.

(iii) Sim 129 and 130 were found to lie on different parts of the bubble moving away from the cluster centre. There is no sign of star formation in Sim 129, while a subset of stars are coincident with Sim 130 in position-velocity space. Furthermore, as the stars in the vicinity of Sim 130 tend to be younger than those in the cluster centre, the stars associated with Sim 130 are likely a new generation of stars formed by feedback from massive stars.

(iv) The geometry of NGC 1893 and the number ratios between old and young groups were inferred from multiple sets of Monte-Carlo simulations. The model adopting the projection angle of $40^{\circ}$ and $n_{\mathrm{og}}: n_{\mathrm{yg}}=2450: 550$ best reproduced the observed RV distribution with a 93 per cent confidence level. The timescale of sequential star formation within the proper distance of 8.9 parsecs was estimated to be $1 \mathrm{Myr}$. The contribution of the newborn stars to the total stellar population in NGC 1893 turned out to be at least 18 per cent.

These results indicate that feedback from massive stars can play an important role in the formation of OB association and supports the self-regulating star formation model (Elmegreen \& Lada 1977). The forthcoming Large Synoptic Survey Telescope will observe the entire regions of a number of $\mathrm{OB}$ associations providing deep photometric data for their members, while the Gaia mission will provide the precise proper motions and distances for many individual stars. Data from such extensive surveys, combined with high resolution spectroscopy, will make unprecedented synergy in uncovering the formation process of $\mathrm{OB}$ associations in the near future.

\section{ACKNOWLEDGEMENTS}

The authors thank the anonymous referee for many useful comments and also thank Perry Berlind, Mike Calkins, and Nelson Caldwell (Smithsonian Astrophysical Observatory) for assisting with Hectochelle observations, Wonseok Kang, Taewoo Kim, and Shinu Jeong (Deokheung Optical Astronomy Observatory) for narrow band imaging observations. This paper has used the data obtained under the K-GMT Science Program (PID: 16A-MMT-001) funded through Korean GMT Project operated by Korea Astronomy and Space Science Institute (KASI). In addition, the data taken from the Harlan J. Smith Telescope at the McDonald Observatory of The University of Texas at Austin (UT), as well as the Discovery Channel Telescope (DCT) at Lowell Observatory were used in this paper. Lowell is a private, non-profit institution dedicated to astrophysical research and public appreciation of astronomy and operates the DCT in partnership with Boston University, the University of Maryland, the University of Toledo, Northern Arizona University and Yale University. This work used the IGRINS that was developed under a collaboration between the UT and the KASI with the financial support of the US National Science Foundation under grant AST-1229522, of the UT, and of the Korean GMT Project of KASI. The resources of SELab were also used in this work. This work was supported by KASI grants 2017186000 and 2017183003. B.L. and H.S. acknowledge the support of the National Research Foundation of Korea, Grant No. NRF-2017R1A6A3A03006413 and NRF2015R1D1A1A01058444, respectively.

\section{REFERENCES}

Ambartsumian, V. A. 1947, In Stellar Evolution and Astrophysics, Armenian Acad. of sci. (German translation, 1951, Abhandl, Sowjetischen Astron., 1, 33.).

André, P., Di Francesco, J., Ward-Thompson, D., et al. 2014, in Protostars and Planets VI, eds. H. Beuther, R. S. Klessen, C. P. Dullemond, \& Th. Henning, 27

Bertout, C. \& Genova, F. 2006, A\&A, 460, 499

Blaauw, A. 1964, ARA\&A, 2, 213

Briceño, C., Preibisch, T., Sherry, W. H., et al. 2007, Protostars and Planets V, 345

Brown A. G. A., Blaauw A., Hoogerwerf R., de Bruijne J. H. J. \& de Zeeuw P. T. 1999, in NATO ASIC Proc. 540: The Origin of Stars and Planetary Systems, ed. C. J. L. N. D. Kylafis, 411

Caramazza, M., Micela, G., Prisinzano, L., et al. 2008, A\&A, 488, 211

Caramazza, M., Micela, G., Prisinzano, L., et al. 2012, A\&A, 539, A74

Castelli, F., \& Kurucz, R. L. 2004, ArXiv Astrophysics e-prints [arXiv:astro-ph/0405087]

Chauhan, N., Pandey, A. K., Ogura, K., et al. 2009, MNRAS, 396,964

Clark, P. C., Bonnell, I. A., Zinnecker, H. \& Bate, M. R. 2005, MNRAS, 359, 21

Copetti, M. V. F., Mallmann, J. A. H., Schmidt, A. A. \& Castañeda, H. O. 2000, A\&A, 357, 621

Dale, J. E., Ercolano, B. \& Bonnell, I. A. 2012, MNRAS, 427, 2852

Dale, J. E., Ercolano, B. \& Bonnell, I. A. 2013, MNRAS, 431, 1062

Dale, J. E., Haworth, T. J. \& Bressert, E. 2015, MNRAS, 450, 1199

Elmegreen, B. G. \& Lada, C. J. 1977, ApJ, 214, 725

Evans, C. J., Kennedy, M. B., Dufton, P. L., et al. 2015, A\&A, 574, A13

Fukuda, N., Hanawa, T. \& Sugitani, K. 2002, ApJ, 568, L127

Getman, K. V., Feigelson, E. D., Garmire, G., Broos, P., \& Wang, J. 2007, ApJ, 654, 316

Gritschneder, M., Naab, T., Burkert, A., et al. 2009a, MNRAS, 393,21

Gritschneder, M., Naab, T., Walch, S., Burkert, A. \& Heitsch, F. 2009b, ApJ, 694, L26

Haworth, T. J., Harries, T. J. \& Acreman, D. M. 2012, MNRAS, 426,203 
Im, M., Ko, J., Cho, Y., et al. 2010, J. Korean Astron. Soc., 43, 75

Kaplan, K. F., Dinerstein, H. L.; Oh, H., et al. 2017, ApJ, 838, 152

Kim, K.-M., Han, I., Valyavin, G. G., et al. 2007, PASP, 119, 1052

Koenig, X. P., Allen, L. E., Gutermuth, R. A., et al. 2008, ApJ, 688,1142

Kroupa, P., Aarseth, S. \& Hurley, J. 2001, MNRAS, 321, 699

Kuhn, M. A., Getman, K. V. \& Feigelson, E. D. 2015, ApJ, 802, 60

Kurtz, M. J. \& Mink, D. J. 1998, PASP, 110, 934

Lanz, T. \& Hubeny, I. 2007, ApJS, 169, 83

Lim, B., Sung, H., Kim, J. S., Bessell, M. S. \& Park, B.-G. 2014, MNRAS, 443, 454

Maheswar, G., Sharma, S., Biman, J. M., Pandey, A. K. \& Bhatt, H. C. 2007 , MNRAS, 379,1237

Markwardt, C. B. 2009, in Astronomical Data Analysis Software and Systems XVIII, eds. D. A. Bohlender, D. Durand, \& P. Dowler, ASP Conf. Ser., 411, 251

Massey, P., Johnson, K. E., \& DeGioia-Eastwood., K. 1995, ApJ, 454, 151

Miller, G. E. \& Scalo, J. M. 1978, PASP, 90, 506

Negueruela, I., Marco, A., Israel, G. L. \& Bernabeu, G. 2007, A\&A, 471, 485

Palla, F. \& Stahler, S. W. ApJ, 581, 1194

Pandey, A. K., Samal, M. R., Chauhan, N., et al. 2013, New Astron., 19, 1

Park, C., Jaffe, D. T., Yuk, I.-S., et al. 2014, Proc. SPIE, 9147, 9147D

Sana, H., de Koter, A., de Mink, S. E., et al. 2013, A\&A, 550, A107

Sharma, S., Pandey, A. K., Ojha, D. K., et al. 2007, MNRAS, 380,1141

Siess, L., Dufour, E., Forestini, M. 2000, A\&A, 358, 5931

Sneden, C. A. 1973, Ph.D. Thesis, The University of Texas at Austin, USA

Sung, H., Bessell, M. S., Chun, M.-Y. 2004, AJ, 128. 1684

Sung, H., Bessell, M. S., Chun, M.-Y., et al. 2017, ApJS, 230, 3

Sung, H., Lim, B., Bessell, M. S., et al. 2013, J. Korean Astron. Soc., 46, 103

Sung, H., Stauffer, J. R., Bessell, M. S. 2009, AJ, 138, 1116

Szentgyorgyi, A., Furesz, G., Cheimets, P., et al. 2011, PASP, 123, 1188

Tonry, J. \& Davis, M. 1979, AJ, 84, 1511

Yuk, I.-S., Jaffe, D. T., Barnes, S., et al. 2010, Proc. SPIE., 7735, 54

Zavagno, A., Russeil, D., Motte, F., et al. 2010, A\&A, 518, L81

This paper has been typeset from a $\mathrm{T}_{\mathrm{E}} \mathrm{X} / \mathrm{LAT}_{\mathrm{E}} \mathrm{X}$ file prepared by the author. 
Table 2. Radial velocities of gas measured from $\mathrm{H}_{2}$ 1-0 S(1) emission line and the forbidden line [N II] $\lambda 6584$.

\begin{tabular}{ccccc}
\hline $\begin{array}{c}\text { R.A. (J2000) } \\
(\mathrm{h}: \mathrm{m}: \mathrm{s})\end{array}$ & $\begin{array}{c}\text { Dec. (J2000) } \\
\left({ }^{\circ}:^{\prime}:^{\prime \prime}\right)\end{array}$ & $\begin{array}{c}V_{\mathrm{LSR}} \\
\left(\mathrm{km} \mathrm{s}^{-1}\right)\end{array}$ & $\begin{array}{c}\epsilon V_{\mathrm{LSR}} \\
\left(\mathrm{km} \mathrm{s}^{-1}\right)\end{array}$ & Remark \\
\hline IGRINS & & & & \\
\hline $05: 22: 54.36$ & $+33: 31: 52.9$ & -10.4 & 0.4 & Sim 129 \\
$05: 22: 54.37$ & $+33: 31: 52.9$ & -10.8 & 0.3 & Sim 129 \\
$05: 22: 54.39$ & $+33: 31: 52.9$ & -11.6 & 0.2 & Sim 129 \\
$05: 23: 07.60$ & $+33: 29: 03.2$ & -2.4 & 0.3 & Sim 130 \\
$05: 23: 07.61$ & $+33: 29: 03.2$ & -2.5 & 0.3 & Sim 130 \\
$05: 23: 07.63$ & $+33: 29: 03.2$ & -2.2 & 0.3 & Sim 130 \\
\hline Hectochelle & & & & \\
\hline $05: 21: 08.82$ & $+33: 22: 58.6$ & -0.7 & 0.7 & \\
$05: 21: 48.41$ & $+33: 21: 08.9$ & -6.6 & 0.4 & \\
\hline
\end{tabular}

The full table is available electronically.

Table 3. Radial velocities of stars.

\begin{tabular}{lccccc}
\hline \hline ID $^{1}$ & $\begin{array}{c}\text { R.A. (J2000) } \\
(\mathrm{h}: \mathrm{m}: \mathrm{s})\end{array}$ & $\begin{array}{c}\text { Dec. }(\mathrm{J} 2000) \\
\left({ }^{\circ}:^{\prime} !^{\prime \prime}\right.\end{array}$ & $\begin{array}{c}V_{\mathrm{LSR}} \\
\left(\mathrm{km} \mathrm{s}^{-1}\right)\end{array}$ & $\begin{array}{c}\epsilon V_{\mathrm{LSR}} \\
\left(\mathrm{km} \mathrm{s}^{-1}\right)\end{array}$ & Remark $^{2}$ \\
\hline BOES & & & & \\
\hline-1 & $05: 22: 46.54$ & $+33: 25: 11.2$ & -2.2 & 3.0 & HD 242935 \\
-2 & $05: 22: 44.00$ & $+33: 26: 26.7$ & -1.9 & 8.6 & BD +33 1025 \\
-6 & $05: 22: 29.30$ & $+33: 30: 50.4$ & -3.8 & 0.8 & HD 242908 \\
\hline Hectochelle 3 & & & & \\
\hline-18 & $05: 23: 12.84$ & $+33: 18: 55.2$ & 2.0 & 1.4 & \\
129 & $05: 22: 05.72$ & $+33: 24: 47.7$ & 15.7 & 1.4 & \\
411 & $05: 22: 11.79$ & $+33: 34: 05.7$ & -38.0 & 0.3 & \\
\hline
\end{tabular}

1 Star IDs are taken from Lim et al. (2014).

2 SB2: Double-lined spectroscopic binary, SB2?: Double-lined spectroscopic binary candidate.

3 The radial velocities of stars with r-statistics larger than 6 .

The full table is available electronically. 